\title{
Design de uma tecnologia mHealth para escores de estratificação de risco cardiovascular apoiado no Letramento em Saúde
}

\author{
Design of an mHealth technology for cardiovascular risk \\ stratification scores supported by Health Literacy
}

Daniele Souto Galeno1, Thereza Maria Magalhães Moreira², Clarice Maria Araújo Chagas

Vergara², Helena Alves de Carvalho Sampaio², José Eurico de Vasconcelos Filho'

DOI: $10.1590 / 0103-1104202012605$

RESUMO Objetivou-se desenvolver um protótipo de design de baixa e alta fidelidade à luz do Letramento em Saúde e contribuir com o profissional de saúde para a escolha do escore de risco cardiovascular mais adequado para cada indivíduo. Estudo metodológico em que se utilizou a metodologia do processo de design de interação participativo centrado no usuário, com contribuições de uma equipe interdisciplinar composta por nutricionista, enfermeira e designer. Contribui-se para a concepção do design de dez telas de baixa fidelidade e de dez telas de alta fidelidade, apoiando-se no checklist, com recomendações para a construção de tecnologias em saúde, considerando-se aspectos fundamentais do Letramento em Saúde. Buscou-se, nos protótipos de baixa e alta fidelidade, desenvolver um layout interativo e agradável para facilitar a utilização pelos profissionais de saúde.

PALAVRAS-CHAVE Aplicativos móveis. Doenças cardiovasculares. Letramento em Saúde. Promoção da saúde.

ABSTRACT The objective was to develop a low and high fidelity design prototype in the light of Health Literacy and to contribute with the health professional to the choice of the most appropriate cardiovascular risk score for each individual. Methodological study using the methodology of the participatory interaction design process centered on the user, with contributions from an interdisciplinary team composed of nutritionist, nurse and designer. Contributes to the design conception of ten low fidelity screens and ten high fidelity screens, based on the checklist, with recommendations for the construction of health technologies, considering fundamental aspects of Health Literacy. We sought, in the low and high fidelity prototypes, to develop an interactive and pleasant layout to facilitate the use by health professionals.

KEYWORDS Mobile applications. Cardiovascular diseases. Health Literacy. Health promotion.

\footnotetext{
1 Universidade de Fortaleza (Unifor) - Fortaleza (CE),

Brasil.

danielegaleno@yahoo.

com.br

2 Universidade Estadual do Ceará (Uece) - Fortaleza (CE), Brasil.
} 


\section{Introdução}

As Doenças Cardiovasculares (DCV) são, atualmente, responsáveis por 7,4 milhões de óbitos no mundo, sendo a principal causa de morte mundial. Estima-se que 17,7 milhões de pessoas morreram acometidas por DCV em 2015, representando $31 \%$ de todos os óbitos no planeta ${ }^{1}$. De acordo com a Organização Mundial da Saúde', as doenças coronarianas, cerebrovasculares, arteriais periféricas, cardíacas reumáticas, além das cardiopatias congênitas, tromboses venosas profundas e embolias pulmonares, são classificadas como doenças do coração e vasos sanguíneos. Os principais fatores de risco para essas doenças são: hiperlipidemia, sedentarismo, hipertensão arterial, tabagismo, consumo nocivo de álcool, diabetes e excesso ponderal1,2.

O envelhecimento da população pode ser considerado como o principal responsável pelo crescente número de óbitos por DCV, revelando a necessidade de investimento em ações de saúde que promovam a prevenção, o controle e o tratamento dessas enfermidades ${ }^{3}$.

Torna-se relevante, portanto, a avaliação do Risco Cardiovascular (RCV) da população pelos profissionais de saúde. Além disso, é por meio da estratificação do RCV que os profissionais de saúde poderão estabelecer metas, buscando incentivar os cidadãos à adesão de um estilo de vida saudável. Para a avaliação do RCV, diversos algoritmos e escores de risco, baseados em estudos com a população, foram elaborados e validados. Conforme a VII Diretriz Brasileira de Hipertensão Arterial4, deve-se evitar a utilização de apenas um escore, pois os fatores de risco considerados pelos instrumentos de estratificação de risco podem variar.

Nas últimas décadas, diversos instrumentos foram desenvolvidos para estimar o risco de desenvolvimento de DCV, como o Escore de Risco de Framingham, a Systematic Coronary Risk Evaluation (Score) e a calculadora de risco QRisk ${ }^{5}$.

O Departamento de Aterosclerose, oficializado como departamento da Sociedade
Brasileira de Cardiologia desde 1994, reconhece oito escores de estratificação de RCV, podendo citar: escores de Framinghan, Reynolds, HeartScore Europe, Prospective Cardiovascular Munster Heart Study (Procam), Assessing Cardiovascular Risk to Scottish Intercollegiate Guidelines Network (Assign), QIntervention ${ }^{\circledR}$, ARIC modificada e Multi-Ethnic Study of Atherosclerosis (Mesa) idade arterial.

As Tecnologias de Informação e Comunicação (TIC) podem facilitar a transmissão de conteúdo aos usuários, pois são responsáveis por captar, transmitir e distribuir informações, por meio de textos, imagens, vídeos ou som ${ }^{6}$. Dentro dessa grande área, está a mobile health (mHealth), definida como a utilização de informações e de tecnologias de comunicação com o intuito de contribuir para a melhoria nos serviços de saúde. A utilização dessas tecnologias pode auxiliar na promoção dos cuidados à saúde, visto que permite o acesso do usuário a dados, além de fornecer um direcionamento sobre tratamento 7 .

Outro ponto positivo desse tipo de tecnologia é a possibilidade de uso pelos profissionais de saúde, permitindo maior agilidade da coleta de informações e transmissão de dados, facilitando a comunicação entre profissional e paciente. Diante disso, há maior contribuição para a adoção de estilo de vida mais saudável, uma vez que o profissional acompanha o paciente pelo monitoramento por mensagens de alerta ${ }^{8}$.

Um estudo revelou que a utilização de aparelhos móveis pelos profissionais de saúde é crescente, alcançando $85 \%$, favorecendo melhoria na assistência à saúde?

Dessa forma, é válido refletir sobre a maneira que essas tecnologias são concebidas, já que as informações sobre saúde contidas nos Aplicativos móveis (App) podem ser de difícil compreensão pela população. Nessa perspectiva, o Letramento em Saúde (LS) apresenta-se como uma ferramenta valiosa, permitindo aos usuários que utilizam essas tecnologias móveis maior compreensão dos conteúdos acessados. Ademais, os App podem 
ser utilizados e acessados por profissionais de saúde a fim de contribuir para a tomada de decisão e elaboração de metas, e as informações de saúde contidas neles podem colaborar também no processo de aprendizagem para futuros profissionais.

O 'Guia para Desenvolvedores e Usuários das Tecnologias mHealth' foi elaborado para orientar a informação em saúde disseminada em websites e App. Diante desse contexto, buscou-se desenvolver um protótipo de design de baixa e alta fidelidade à luz do LS pautado no direcionamento proposto pelo Guia existente ${ }^{10}$. A intenção é contribuir com o profissional de saúde para a escolha do escore de RCV mais adequado para cada indivíduo.

\section{Material e métodos}

Estudo metodológico, que propôs a concepção do design do aplicativo Calculadora de Estratificação de Risco Cardiovascular (Cerc). Para isso, é fundamental a busca por aplicativos existentes envolvendo essa temática para identificação das necessidades em uma avaliação comparativa (Benchmarking). O processo de Benchmarking caracteriza-se pela determinação do objeto, identificação de parceiros, coleta e análise de dados, definição das metas, implementações das ações e monitoramento dos resultados encontrados"1. Foi realizada uma busca com a palavra 'risco cardiovascular' nos dois maiores sistemas operacionais portáteis existentes na atualidade, Android e IOS, nas lojas virtuais Play Store ${ }^{\circledR}$ e App Store $^{\circledR}$.

Para melhor compreensão, esse processo foi dividido em quatro etapas descritas a seguir: $1^{\text {a }}$ etapa: levantamento nas lojas virtuais Google Play Store ${ }^{\circledR}$ e App Store ${ }^{\circledR}$ com a palavra 'risco cardiovascular'; $2^{\text {a }}$ etapa: seleção dos App com foco no cálculo de RCV; $3^{\text {a }}$ etapa: exclusão dos aplicativos pagos e em línguas distintas do português brasileiro; $4^{\mathrm{a}}$ etapa: número final de App selecionados, conforme os critérios de inclusão.

Para a construção do protótipo de design da tecnologia mHealth, utilizou-se a metodologia do processo de Design de Interação Participativo Centrado no Usuário. Esse modelo possibilita o desenvolvimento de produtos interativos fáceis, eficientes e agradáveis de usar. Quatro aspectos básicos foram considerados para o processo do design: estabelecimento de requisitos por meio da identificação das necessidades; elaboração de alternativas de design; concepção do protótipo; e avaliação da versão interativa, estes devem complementar um ao outro ${ }^{\mathbf{1 2}}$.

O estabelecimento dos requisitos básicos a serem considerados no design do aplicativo ocorreu após reuniões de brainstorming, no intuito de identificar as funções que deveriam ser contempladas pelo protótipo do sistema. Para estabelecer a prioridade dos requisitos, foram adotadas as denominações sugeridas pelos pesquisadores 'essencial', 'importante' e 'desejável'. Após essa etapa, foram elaborados os requisitos funcionais da ferramenta apresentados a seguir:

- Cadastro no aplicativo: o aplicativo deveria permitir que o profissional realizasse seu cadastro em uma opção na tela inicial. Para isso, os seguintes campos deveriam ser preenchidos: nome, sobrenome, número do conselho de classe, como identificador de login (obrigatório); e-mail (obrigatório), senha (obrigatório); confirmação da senha (obrigatório);

- Acesso ao sistema: o sistema deveria permitir que o profissional realizasse login no aplicativo por meio dos dados de cadastro, sendo solicitado o número do conselho e a senha;

- Recuperação de senha: na tela inicial de acesso, o sistema deveria oferecer a funcionalidade de recuperação de senha da conta do profissional via e-mail cadastrado;

- Cadastro clínico do paciente (escore de $\mathrm{RCV}$ ): o aplicativo permitiria ao profissional logado cadastrar as características de saúde 
dos pacientes/usuários sob seus cuidados a fim de associá-los a um perfil clínico, possibilitando a escolha de um tipo de Escore de RCV de acordo com os dados disponíveis para cálculo. O profissional poderia escolher o melhor instrumento a ser utilizado de acordo com os fatores de risco apresentados pelo paciente. Os Escores de Risco RCV considerados no estudo foram: Framingham, Reynolds, HeartScore Europe, Procam, ASSIGN, QRisk 2, ARIC modificada e a Estratificação de risco, pois são reconhecidas pela VII Diretrizes Brasileiras de Hipertensão;

- Perfil: o sistema permitiria a edição dos seguintes dados de perfil: nome, sobrenome, e-mail e senha;

- Acompanhamento do paciente: com base nas informações do cadastro clínico do paciente/usuário, o profissional receberia informações sobre qual o resultado do cálculo de estratificação de RCV específico para seu perfil, bem como o escore considerado pelo profissional, podendo editar os fatores de risco a qualquer momento;

- Informações gerais: o aplicativo deveria possuir uma área contendo informações sobre: notícias e eventos relativos à saúde, 0 profissional poderia compartilhar as informações com seus alunos e outros profissionais.

Após a definição desses requisitos, a segunda fase contemplou os processos de design e redesign, ou seja, a definição de como seriam as telas do aplicativo. O processo de design ocorreu por meio da criação do protótipo de baixa fidelidade, caracterizado por não apresentar recursos de interação ${ }^{\mathbf{1 3}}$. Para a construção do protótipo de baixa fidelidade, dez telas foram desenvolvidas. Nessa etapa, houve a contribuição de profissionais da saúde - médico, enfermeira e nutricionista - com o profissional de designer para a concepção das telas e interfaces, considerando o checklist proposto por Eichner e Dullabh ${ }^{\mathbf{1 0}}$ com recomendações para a construção de tecnologias em saúde considerando aspectos fundamentas do LS. O checklist enfoca quatro domínios: linguagem, conteúdo, formato e aspectos culturais. Cada bloco de domínio apresenta itens específicos que devem ser atendidos.

Já na construção da versão interativa, elaborou-se o protótipo de alta fidelidade, contemplando, além do protótipo de baixa fidelidade, tipografia, iconografia e paleta de cores, gerando dez telas interativas. Para a construção do design do protótipo de baixa fidelidade e alta fidelidade, utilizou-se a ferramenta Adobe Photoshop ${ }^{\circledR}$.

O estudo descreve a concepção do design de um aplicativo, mas existe a pretensão de idealização do desenvolvimento do aplicativo móvel.

A presente pesquisa seguiu a Resolução $n^{0} 466 / 201214$, que rege pesquisas com seres humanos, e foi aprovada pelo Comitê de Ética em Pesquisas com Seres Humanos da Universidade Estadual do Ceará (Uece), sob número CAAE 69459317.0.0000.5534, no projeto guarda-chuva 'Plano Conecta Saúde: aliando inovação tecnológica e letramento em saúde na luta contra as DCNT'.

\section{Resultados e discussão}

O quantitativo de aplicativos para dispositivos móveis voltados a RCV disponível na plataforma tecnológica Google Play Store ${ }^{\circledast}$ foi: $1^{\text {a }}$ etapa, $n=250 ; 2^{\text {a }}$ etapa, $n=53$; $3^{\text {a }}$ etapa, $n=50$; $4^{\mathrm{a}}$ etapa, $\mathrm{n}=3$. Já na plataforma tecnológica App Store ${ }^{\circledR}$, encontrou-se: $1^{\mathrm{a}}$ etapa, $\mathrm{n}=293$; $2^{\mathrm{a}}$ etapa, $\mathrm{n}=26 ; 3^{\mathrm{a}}$ etapa, $\mathrm{n}=22 ; 4^{\mathrm{a}}$ etapa, $\mathrm{n}=4$.

Uma constatação, logo de início, é o baixo número de App voltados à temática RCV que atendem aos critérios de inclusão, o que reforça a necessidade da concepção do aplicativo proposto pelo estudo. Ademais, das tecnologias móveis incluídas na avaliação comparativa, nenhuma apresentou um compilado dos escores de estratificação de RCV.

Dados de uma pesquisa que propôs a identificação da tendência de pesquisas no Brasil 
que utilizam tecnologia móvel na área da saúde revelou que, dos 27 artigos incluídos no estudo - alguns sobre concepção de aplicativos para identificação de melanomas, acesso para dados clínicos de pacientes hospitalizados, aplicativo sobre vacinação no Brasil, entre outros -, nenhum envolvia a concepção de uma tecnologia móvel voltada ao cálculo para estratificação de RCV ${ }^{15}$.

Outro estudo 16 investigou artigos que discutem o design, desenvolvimento e avaliação de App para profissionais de saúde, estudantes de medicina, enfermagem e pacientes. Nele, 83 aplicativos foram encontrados, sendo 57 destinados a uso médico, 11 projetados para estudantes de medicina ou enfermagem e 15 voltados ao paciente. Oito App foram identificados como calculadora médica, contemplando assuntos diversos, como: queimadura corporal, dosagem de medicamentos, fórmulas médicas, entre outros.

O design do aplicativo Cerc proposto neste estudo buscou ofertar para os profissionais de saúde, que acompanham indivíduos acometidos por doenças crônicas, uma tecnologia que apresentasse um compilado de escores de estratificação de RCV validados, a fim de contribuir e apoiar os profissionais na tomada de decisão com a possibilidade de identificar precocemente o risco de acometimento por essas doenças.

A pesquisa considerou as percepções e experiências da equipe interdisciplinar (nutrição, medicina, enfermagem e designer em tecnologia) sobre informações relacionadas com a saúde e desenvolvimento de tecnologia mHealth específico para esse problema.

A primeira etapa de construção do protótipo de baixa fidelidade gerou dez telas (figuras 1 e 2). Nela, buscou-se contemplar requisitos fundamentais para a correta avaliação por meio dos escores de estratificação de risco selecionados, além da utilização do checklist na intenção de contemplar o máximo de aspectos de cada domínio, como: escrita em voz ativa, informações relevantes para os usuários, texto escuro escrito em fundo de tela claro, estilo e tamanho da fonte consistente ao longo do texto, utilização de letras maiúsculas e minúsculas, margem dos textos justificadas à esquerda, entre outros.

Figura 1. Telas de cadastro do usuário do protótipo de baixa fidelidade
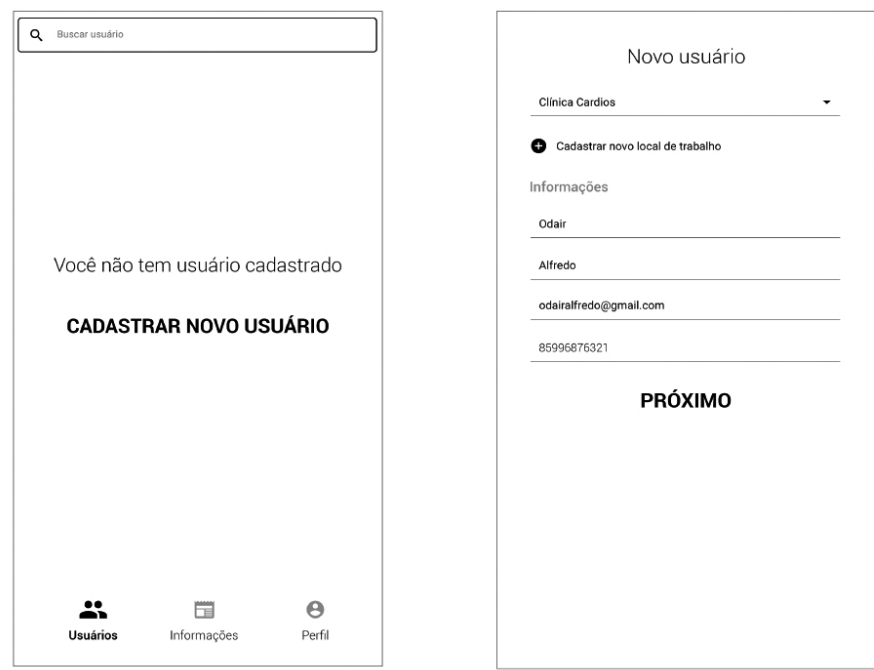
Figura 2. Telas de fatores de risco consideradas pelos escores de estratificação de risco do protótipo de baixa fidelidade

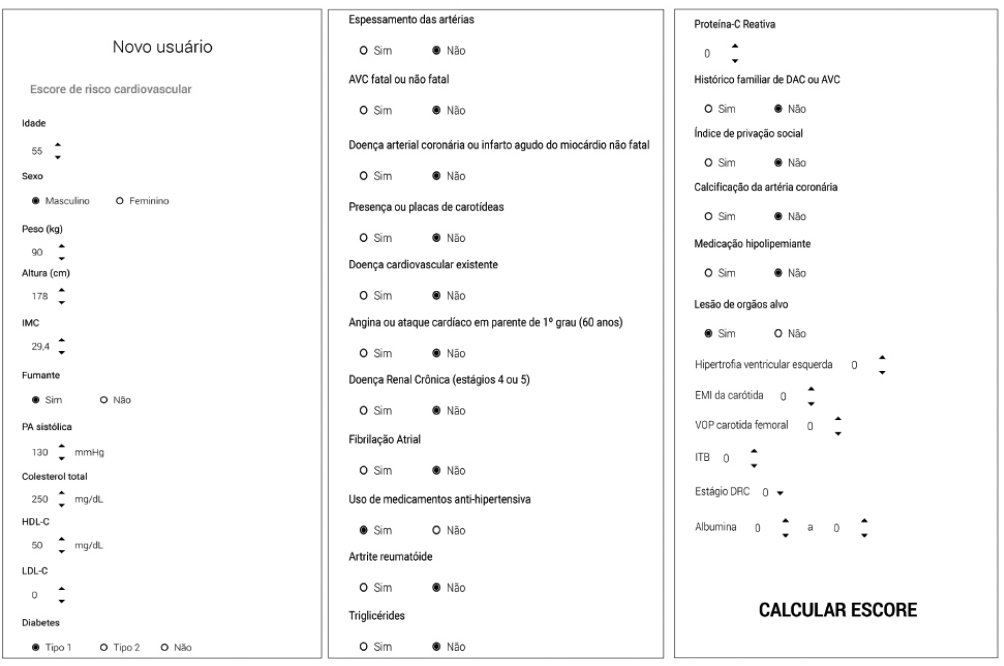

Fonte: Elaboração própria.

Após avaliação pela equipe interdisciplinar das telas do protótipo de baixa fidelidade, percebeu-se a necessidade de alguns ajustes relevantes, como a presença do botão de retorno nas telas, contemplando um dos aspectos propostos pelo checklist, inclusão da resposta 'dado não fornecido', considerando que possivelmente os profissionais não tenham acesso a todas as informações no momento da avaliação.

Diante disso, a fase seguinte deteve-se à concepção do protótipo de alta fidelidade com o desenvolvimento de dez telas (figuras 3 a 5 ).

Figura 3. Telas de cadastro do usuário do protótipo de alta fidelidade
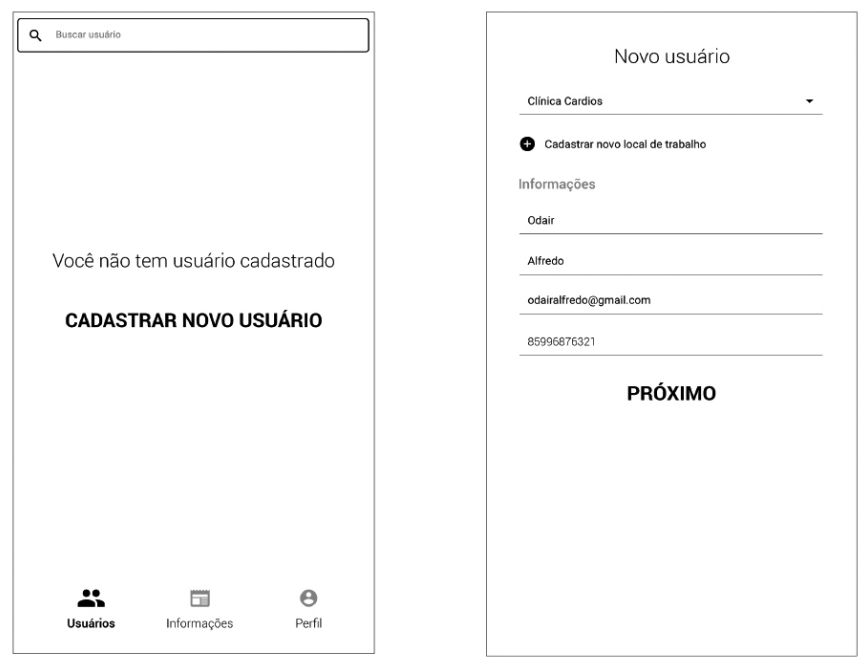
Figura 4. Telas de fatores de risco consideradas pelos escores de estratificação de risco do protótipo de alta fidelidade
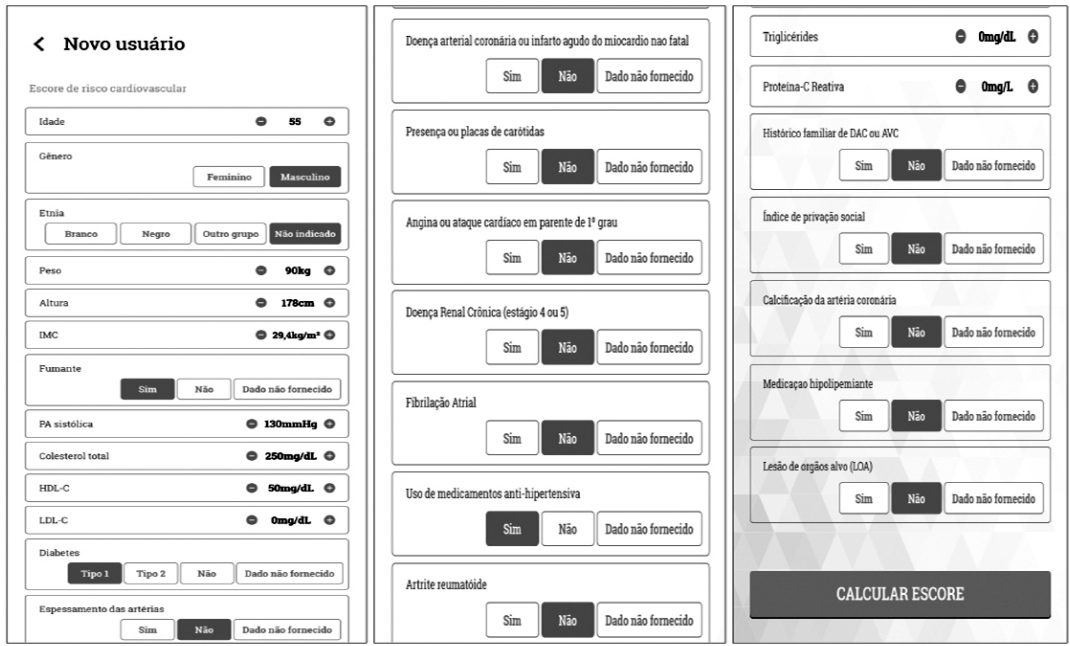

Fonte: Elaboração própria

Figura 5. Telas de resultado pelos escores de estratificação de risco do protótipo de alta fidelidade
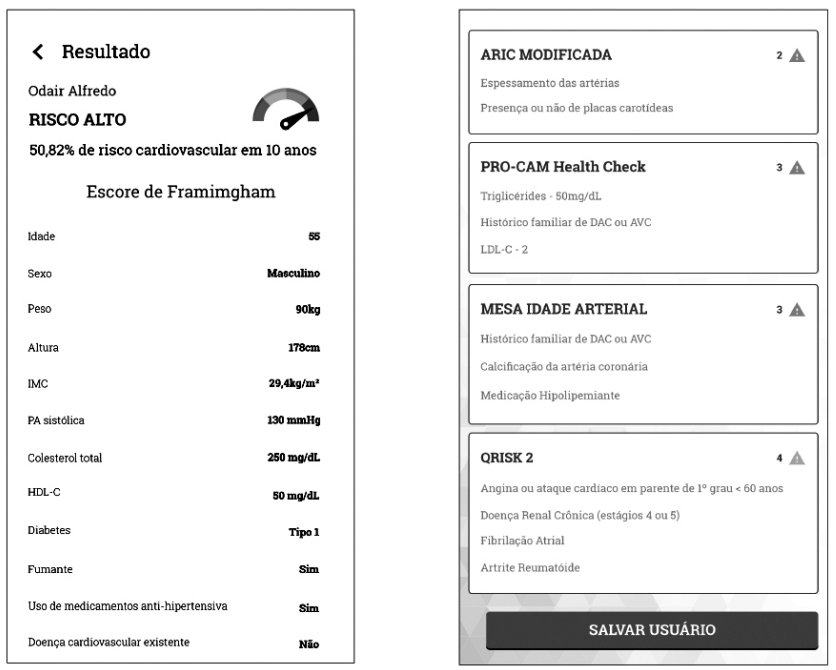

Fonte: Elaboração própria.

O design do protótipo do aplicativo desenvolvido buscou contribuir para a identificação precoce do risco para o desenvolvimento de DCV, oferecendo ao profissional uma tecnologia contendo diferentes Escores de RCV, possibilitando a escolha conforme os fatores de risco apresentados pelos pacientes e usuários. A construção interdisciplinar contribuiu para elaboração de um produto que buscou contemplar diversos olhares dos diferentes campos do saber. 
A participação da equipe interdisciplinar no processo de construção do design do protótipo do aplicativo melhora a definição das etapas e avalia melhor os caminhos para alcançar o objetivo proposto. Os autores defendem ainda que o processo metodológico centrado no protótipo possibilita o design mais criativo, ampliando os ciclos interativos por meio das correções realizadas em cada fase ${ }^{17}$.

Acredita-se que o design do protótipo do aplicativo proposto neste trabalho poderá contribuir também para o ensino superior na área da saúde, pois o conteúdo ofertado por tecnologias de informação e comunicação é apresentado de forma lúdica e interativa, possibilitando que o professor compartilhe informações ligadas à temática com seus alunos, para que o processo ensino-aprendizagem possa ser mais prazeroso e prático.

Os App de uso médico tornam as informações em saúde acessíveis de qualquer lugar. As calculadoras médicas oferecem, além de informações, oportunidades de aprendizagem móvel para estudantes da área da saúde ${ }^{\mathbf{1 8}}$. Alguns autores ${ }^{19}$ defendem que esse tipo de aplicativo deve ser utilizado especialmente na atenção primária. Muitos aplicativos em saúde estão disponíveis nas lojas virtuais, no entanto, muitos deles não foram discutidos na literatura se apresentam respaldo científico ${ }^{\mathbf{1 6}}$.

É preciso ampliar o olhar aos protótipos voltados à área da saúde para além de uma simples ferramenta, compreendendo que estes podem contribuir para o levantamento de parâmetros com usuários, além da realização de avaliações para sucesso do produto ${ }^{17}$.

As telas apresentadas na figura 4 foram divididas em três apenas para apresentação, pois, na proposta do design, as informações são apresentadas em apenas uma tela, evitando que o profissional necessite utilizar o botão de voltar caso não se lembre de algum valor registrado. Considera-se que a necessidade apenas de 'deslizar' o botão de rolagem em uma única tela facilite a visualização de todas as informações, mesmo que o profissional sinta necessidade de editar alguma informação anterior. Apesar de apresentarem muitas informações, estas são necessárias, pois são os diferentes fatores de risco considerados pelos Escores de RCV, possibilitando que o profissional, no momento da avaliação, selecione de forma rápida as respostas de cada fator de risco.

Acredita-se que a concepção do design do protótipo do aplicativo proposto, apoiado em alguns aspectos do checklist ${ }^{10}$, poderá contribuir para apresentação das informações aos profissionais de saúde de forma mais clara, pois os quesitos do checklist foram respeitados.

Os termos utilizados em cada bloco de domínio foi descrito atendendo às capacidades de entendimento de cada público, seja ele do usuário ou profissional de saúde, segundo o LS. Algumas terminologias comuns à área dos profissionais de saúde que lidam com as DCV foram também inseridas.

\section{Conclusões}

O design do protótipo do aplicativo proposto desenvolvido buscou contribuir com informações relevantes sobre o RCV, oferecendo apoio para a tomada de decisão pelos profissionais de saúde, além de considerar o LS, pois é imprescindível que esse tipo de tecnologia seja concebida apoiada nesses princípios.

Outro diferencial da proposta foi a inclusão de diferentes escores de estratificação de RCV para que o profissional de saúde possa escolher o mais adequado de acordo com os fatores de risco apresentados pelos usuários.

Ante o exposto, destaca-se a necessidade de desenvolver requisitos e funções que sejam relevantes para a temática, com participação interdisciplinar na construção do design do protótipo do aplicativo buscando diferentes olhares. A concepção desse tipo de tecnologia apresenta-se também como ferramenta de apoio ao ensino, contribuindo para o processo de formação profissional. Buscou-se, nos protótipos de baixa e alta fidelidade, desenvolver um layout interativo e agradável para facilitar a utilização pelos profissionais de saúde. 


\section{Colaboradores}

Galeno DS (0000-0002-3976-7271)*, Moreira

TMM (0000-0003-1424-0649)*, Vergara

CMAC (0000-0003-1709-9951)*, Sampaio
HAC (0000-0001-5353-8259)*, Vasconcelos Filho JE (0000-0002-6881-0814)*, contribuíram igualmente para a elaboração do manuscrito.

\section{Referências}

1. Organização Mundial da Saúde. Determinantes Sociais e Riscos para a Saúde, Doenças Crônicas não transmissíveis e Saúde Mental [internet]. [acesso em 2018 jun 11]. Disponível em: https://www.paho.org/ bra/index.php?option=com $\_$content\&view $=$article\& $\mathrm{id}=5253$ : doencas-cardiovasculares $\&$ Itemid $=839$.

2. Brasil. Ministério da Saúde, Secretaria de Vigilância em Saúde. Vigitel Brasil 2016: vigilância de fatores de risco e proteção para doenças crônicas por inquérito telefônico: estimativas sobre frequência e distribuição sociodemográfica de fatores de risco e proteção para doenças crônicas nas capitais dos 26 estados brasileiros e no Distrito Federal em 2016. Brasília, DF: Ministério da Saúde; 2017.

3. Brant LCC, Nascimento BRP, Valéria MA, et al. Variações e diferenciais da mortalidade por doença cardiovascular no Brasil e em seus estados, em 1990 e 2015: estimativas do Estudo Carga Global de Doença. Rev. bras. epidemiol. [internet]. 2017 [acesso em 2018 nov 21]; 20(supl):116-128. Disponível em: http:// www.scielo.br/scielo.php?script=sci_arttext\&pid=S 1415790X2017000500116\&lng=en.

*Orcid (Open Researcher and Contributor ID).
4. Sociedade Brasileira de Cardiologia. $7^{\mathrm{a}}$ Diretrizes Brasileiras de Hipertensão Arterial - VII DBH. Rev.
Soc. Bras. Hipert. 2016; 107(supl3):1-103.

5. Damen JA, Hooft L, Schuit E, et al. Prediction models for cardiovascular disease risk in the general population: systematic review. BMJ. 2016; (353):i2416.

6. Veloso FC. Informática: conceitos básicos. 10. ed. Rio de Janeiro: Elsevier; 2017.

7. Rocha FS, Santana EB, Silva ES, et al. Uso de Apps para a promoção dos cuidados à saúde. In: Anais III Seminário de Tecnologias Aplicadas em Educação e Saúde - STAES; 8-9 maio 2017; Salvador (BA): Universidade do Estado da Bahia; 2017.

8. Mann S. Wearable computing [internet]. In: The Interaction Design Foundation. The encyclopedia of human-computer interaction. 2. ed. Aarhus: Denmark: The Interaction Design Foundation; 2014 [acesso em 2018 jun 20]. Disponível em: https://www.interaction-design.org/encyclopedia/wearable_computing.html.

9. Oliveira TR, Costa FMR. Desenvolvimento de aplicativo móvel de referência sobre a vacinação do Brasil. J. Health Inform. [internet]. 2012 [acesso em 2018 out 10]; 4(1):23-7. Disponível em: http://www.jhi-sbis. 
saude.ws/ojs-jhi/index.php/jhi-sbis/article/viewFile/161/109.

10. Eichner J, Dullabh P. Accessible Health Information Technology (Health IT) for Populations With Limited Literacy: A Guide for Developers and Purchasers of Health IT [internet]. Rockville: Agency for Healthcare Research and Quality; 2007. [acesso em 2018 out 10]. Disponível em: https://digital.ahrq.gov/sites/ default/files/docs/page/LiteracyGuide_0.pdf.

11. Melo AM, Carpinetti LC, Silva WT. Proposta de Metodologia para Identificação de Objeto de Estudo de Benchmarking. São Paulo: ENEGEP; 2000.

12. Preece J, Rogers Y, Sharp H. Design de interação: além de interação homem-computador. 3. ed. Porto Alegre: Bookman; 2013.

13. Santos RLG. Usabilidade de interfaces para sistemas de recuperação de informação na web: estudo de caso de bibliotecas on-line de universidades federais brasileiras. [tese] [internet]. Rio de Janeiro: Pontifícia Universidade Católica do Rio de Janeiro; 2006. 347 f. [acesso em 2018 mar 3]. Disponível em: http://www2.dbd.pucRio.br/pergamum/tesesabertas/0313143_06_cap_10.pdf.

14. Conselho Nacional de Saúde. Resolução no 466, de 12 de dezembro de 2012. Aprova normas regulamentadoras de pesquisas envolvendo seres humanos [in- ternet]. Diário Oficial da União. 13 Dez 2013. [acesso em 2018 mar 3]. Disponível em: http://conselho. saude.gov.br/resolucoes/2012/Reso466.pdf.

15. Tibes CMS. Aplicativo móvel para prevenção e classificação de úlceras por pressão. [dissertação]. São Carlos: Universidade Federal de São Carlos; 2014. $134 \mathrm{p}$.

16. Mosa ASM, Yoo I, Sheets L. A systematic review of healthcare applications for smartphones. BMC Med. Info. Decis. Making. 2012; 12(67):1-31.

17. Oliveira LMR, Vergara CMAC, Sampaio HAC, et al. Tecnologia mHealth na prevenção e no controle de obesidade na perspectiva do letramento em saúde: Lisa Obesidade. Saúde debate. 2018; 42(118):714-723.

18. Cibulka NJ, Crane-Wider L. Introducing Personal Digital Assistants to Enhance Nursing Education in Undergraduate and Graduate Nursing Programs. J Nurs Educ. 2010; 50:115-118.

19. Ordúñez P, Tajer C. Disseminating cardiovascular disease risk assessment with a PAHO mobile app: a public eHealth intervention. Rev Panam Salud Publica. 2015; (38):82-5.

\footnotetext{
Recebido em 07/02/2019

Aprovado em 07/06/2020

Conflito de interesses: inexistente
}

Suporte financeiro: não houve 